\title{
Nanophotonics for optical super resolution from an information theoretical perspective: a review
}

\author{
Zeev Zalevsky, ${ }^{\text {a }}$ Vicente Mico, ${ }^{\text {b }}$ and Javier Garciab \\ ${ }^{a}$ School of Engineering, Institute of Nanotechnology, Bar-Ilan University, \\ Ramat-Gan 52900, Israel \\ zalevsz@eng.biu.ac.il \\ ${ }^{\mathrm{b}}$ Departamento de Óptica, Universitat de Valencia, c/Doctor Moliner 50, 46100 Burjassot, \\ Valencia, Spain
}

\begin{abstract}
Optical super resolution is one of the most applicable as well as scientifically exciting field of optics dealing with imaging and aiming to improve the spatial resolvable capabilities of any imaging system (digital or ophthalmic) without modifying the optical parameters of the imaging lenses or the geometry of the detection array. Recent developments in nanotechnological fabrication capabilities open new doors of possibilities in fulfilling modified and improved super resolving configurations.

Fundamental concepts for obtaining super resolved imaging are related with time, angular, wavelength, polarization and gray levels multiplexing. The state of the art in the field of optical super resolution is reviewed while showing its relation with nanophotonics.
\end{abstract}

Keywords: resolution, imaging systems, Fourier optics, image reconstruction.

\section{INTRODUCTION}

Every imaging system has limited resolving capability which is expressed in terms of the minimal distance that two infinitely small spatial features can be positioned near each other and yet being seen as two separable items [1]. This resolving capability is limited by several factors.

The first one is related to diffraction [2]. The maximal angle of diffraction generated from a given feature is linearly proportional to the optical wavelength and inversely proportional to the size of that feature. Only the angles arriving within the diameter of the imaging lens are imaged on top of the detection plane. Mathematically this limitation means that the smallest resolvable feature is proportional to the product between the optical wavelength and the $\mathrm{F}$ number of the imaging lens (the ratio between its focal length and its diameter). And conceptually it means that the object's spectrum is trimmed by the limited aperture of the imaging system and, thus, the achievable resolution becomes also restricted.

The second limitation is related to the geometry of the detection array. This limitation can be divided into two types of constrains. The first type is related to the number of the sampling points and the distance between such two adjacent points. The denser the 2D spatial sampling grid is, the better is the quality of the sampled image. The second type of constrain is related to the spatial responsivity of each sampling pixel. Since each pixel integrates the light that impinges on its area, the 2D sampling array is not an ideal sampling array. This local spatial integration done at each pixel results with low pass filtered image [3].

The third limitation is related to the dynamic range of the pixels, its noises and the number of quantization bits that are used for the analogue to digital conversion of the electrons collected in the electronic capacitor of every sampling pixel [1].

Is there a way to overcome the above mentioned limitations? Assuming that the number of degrees of freedom contained within the signal is smaller than the overall number that may be transmitted through the optical system the answer is yes given that proper adaptation process is applied [4-7]. Note that the proposed theorem is general but when imaging is concerned by the term of signal we refer to 2-D image. One needs to 
remember that in order to see better we need to resolve spatial information. However the imaging system can transfer information that is contained in multi dimensional hyper space including axes such as space, time, coherence, polarization, color, dynamic range etc. Each one of those axes can be used in order to convert the non resolved spatial degrees of freedom, to transmit them through the band limited imaging system and then to back-convert them into the space domain yielding super resolved image. Thus, the basic principle is to do encoding of the spatial information prior to its transmission through the imaging system and then a decoding procedure for the reconstruction. The domain that is used for the conversion determines the type of information multiplexing that is applied in the system. The idea is always to realize this adaptation and multiplexing without changing the optical parameters of the imaging system. The optical approaches that do this involve addition of special optical elements and sometimes some digital image post processing. But all of them have the same meaning: the special encoding element is used to divert on-axis the high frequency diffracted bands of the object's spectrum towards the system's aperture, and the remaining problem is how to recover and replace such additional high spatial-frequency information into its original position at the object's spectrum in order to achieve the super resolved image.

The encoding of high frequency spatial information is usually done by either placing a physical element as grating or by projecting the encoding pattern. The obtained resolution improvement in grating related approaches will be limited by the smallest feature in the grating. However such a grating occupied less volume than a high quality lens objective having equivalent NA and thus in microscopy applications where volume constrains are crucial the usage of such super resolving technique can be beneficial. Except of the gain in volume, the addition or projection of encoding pattern (e.g. the grating) together with low NA objective is less expensive than using only a high NA objective. In the configuration where instead of physical encoding mask the pattern is projected, the constrains are the same. In this case the high resolution details are NOT projected by using high NA lens but rather using e.g. a simple diffuser (e.g. when speckles pattern is being projected for encoding) or by interference of several point sources that generate the desired encoding pattern over the inspected object. Therefore in this case as well, the resolution enhancement is obtained without using high quality, high cost and high volume lenses with large NA. The projection of the encoding pattern is important in imaging of remote objects as in security related applications.

Another benefit of the super resolution approach is the capability to obtain high transversal resolution enhancement and yet to preserve significant depth of focus capabilities. Regular high NA lens has small depth of focus. Nevertheless, the resolution enhancement come on the extent of energetic efficiency since only a portion of the energy, which is diverted by the encoding grating to the various diffraction orders, is captured by the low NA lens. Therefore the synthetic aperture generation is energetically inefficient.

In Sec. 2 we review the diffraction related super resolving approaches. In Sec. 3 we focus on the geometrical super resolution field and in Sec. 4 we present methods to extend the depth of focus (axial resolution). The paper is concluded in Sec. 5.

\section{DIFFRACTIVE OPTICAL SUPER RESOLUTION}

In this Sec. we will review existing super resolving approaches aiming to overcome the limitations posed by the $\mathrm{F}$ number of the imaging lens. As previously mentioned the minimal resolvable distance due to diffraction equals to:

$$
\delta=1.22 \lambda \frac{F}{D}
$$

where $\lambda$ is the optical wavelength, $F$ the focal length of the imaging lens and $D$ is the diameter of its aperture. 


\subsection{Single path passive approaches}

\subsubsection{Time multiplexing}

One of the most popular directions for super resolution is related to usage of the time domain in order to multiplex the relevant spatial degrees of freedom that before could not pass through the aperture of the imaging lens.

The basic configuration was first introduced by Francon [8] and later on by Lukosz [9] as it is presented in Fig. 1. It includes usage of two gratings attached to the object (encoding grating) as well as to the image (decoding grating). Both are moving in opposite directions (for imaging system with negative magnification factor). The imaging system presented in Fig. 1 is a 2-F system with magnification of -1 (obviously this approach is general and it is not limited to a certain value of magnification). The encoding grating generates spectral replications such that the high frequencies, previously cut off by the limited aperture of the imaging lens, are now folded inside it. The movement generates different Doppler shift per each replication, i.e. each one of the replications has differently time varying phase factor in the spatial spectrum domain. Thus, although all of the replications were folded inside the aperture and were mixed together, they can still be separated after passing through the aperture while reconstructing the original spectrum.

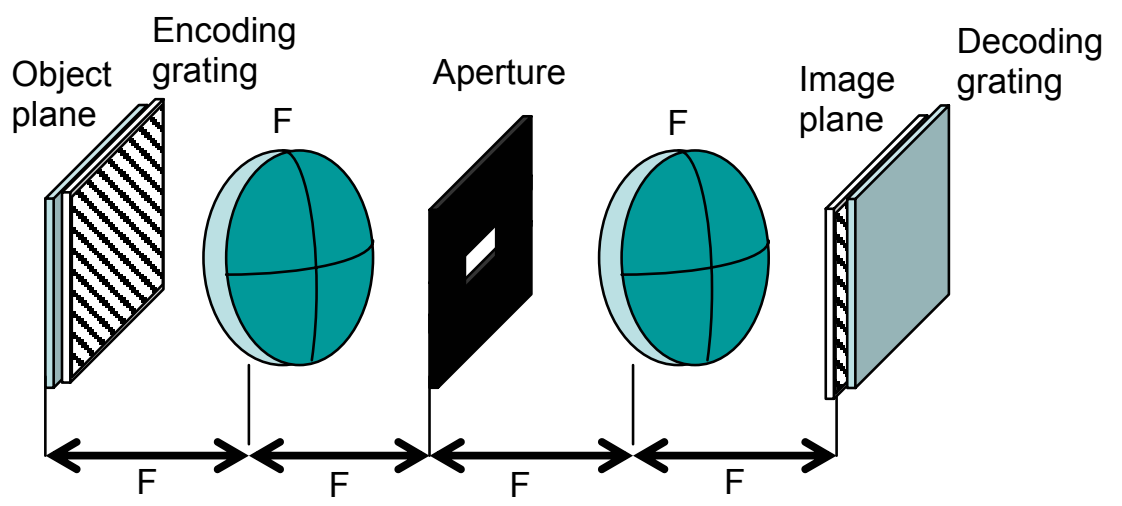

Fig. 1. Time multiplexing basic approach.

The spectrum reconstruction is done by the decoding grating which once again replicates the folded spectral slots (with the different Doppler shifts) while generating another and opposite Doppler shift. In other words, the decoding grating will generate opposite time varying phase factor for the spectral replications. Only the correct spectral information will have correction of the Doppler shift. For the other non relevant information cross terms a certain Doppler shift will still remain and thus after time averaging those undesired cross terms will be averaged to zero [10] (i.e. the decoding grating generated opposite time varying phase factor that will cancels the original temporal phase only in the proper spectral slots and therefore after time averaging all the undesired temporal slots will be averaged to zero).

An extension of this approach for 2D was demonstrated in Refs. 11 and 12. This approach was simplified by usage of digital rather than physical decoding grating [13] and later on by usage of projected encoding grating rather than a physical grating that is attached to the object [14-16]. The same approach was demonstrated also with random (rather than periodic) encoding/decoding patterns. For instance, one applicable technique was realized by projecting speckle pattern on the object and using the same random pattern in digital post processing in order to decode and to reconstruct the high resolution image [17-21].

Following that similar concept was developed for the case of scenarios where relative movement exists between the object and the background [22] or between rain 
droplets [23] or turbulence [24] and the object. In all of those cases the background, the droplets or the turbulence are used as the random encoding function generating Doppler shifts to the spatial information of the object due to its relative movement in comparison to the inspected object.

In all of those approaches the improvement of resolution is as good as our a priori information regarding the encoding distribution. The smallest its features are the more improvement we will be able to obtain in resolving the object. In the case of rain droplets and for the scenario where the droplets are sparse, one can avoid having the a priori information since in this case the encoding distribution can be extracted from the set of captured images themselves just by applying proper digital processing algorithm [23]. But here as well the maximal super resolving improvement will provide reconstructions of features only as small as the smallest droplet.

Note that an interesting extension of the time multiplexing approach is related to the usage of light's coherence domain for the encoding of the spatial information. Coherence is related to fast temporal variations of the phase of the optical wave front and thus basically it is a time multiplexing related approach. In the coherence case one is synthesizing the illumination source to have desired spatial transversal or axial (i.e. temporal) coherence such that when illuminating the object, its different spatial regions can be encoded with orthogonal temporal phase variations such that even after their spatial mixture can later on be recovered and reconstructed $[25,26]$. In this approach as before one needs to know the encoding and the decoding architecture, the difference with the regular time multiplexing technique is in the required temporal averaging period after which proper resolution reconstruction may be obtained.

\subsubsection{Angular multiplexing}

The fundamentals of this approach were first presented by Lukosz [27]. In this case once again two gratings are used but no movement is required. All the components of the optical system are static. The encoding and decoding is done by using the field of view. If a priori we know that the object is field of view limited [28], the rest of the field of view may be used to improve the imaging resolution of our system. The encoding grating generates replications of the object while each replication contains different spectral information. The decoding grating super imposes the replications one over the other while only in the central replication all spectral slots can be properly super imposed [29].

The basic setup for this case may be seen in Fig. 2. Here both gratings are positioned without physical contact with the object or the imaging sensor. However, the condition to obtain super resolution is $\mathrm{Z}_{1}=\mathrm{Z}_{2}$ which means that not the two of them are positioned in the volume which is between the object and the image plane. Only one of them is positioned in the desired spacing while the other one is left outside. In order to solve this drawback a modified approach including three gratings was proposed $[30,31]$. There all the three gratings can be allocated within the space between the object and the image plane.

The fact that one needs to sacrifice the field of view is also somewhat problematic. One possible solution with this drawback deals with the case that the positions of the ghost images (e.g. the undesired replications) are wavelength dependent. Thus, in the case that white light illumination is used all undesired replications are averaged (because there positions are varied across the field of view) while the central replication (i.e. the zero order whose spatial location is not wavelength dependent) which is properly super imposed is strengthened [32]. Thus, in this case actually the payment in the field of view is converted to payment in the dynamic range since the reconstructed high resolution image appears on top of an averaged background level.

Note that here, as in the time multiplexing approaches previously mentioned, the resolution improvement will correspond to the smallest feature in the encoding grating (the diffraction angle it generates). If an imaging lens with larger NA, that corresponds to the diffraction angle created by the grating, will be used similar resolving results will be obtained. Nevertheless using the grating instead of a lens with larger NA saves volume and costs. 


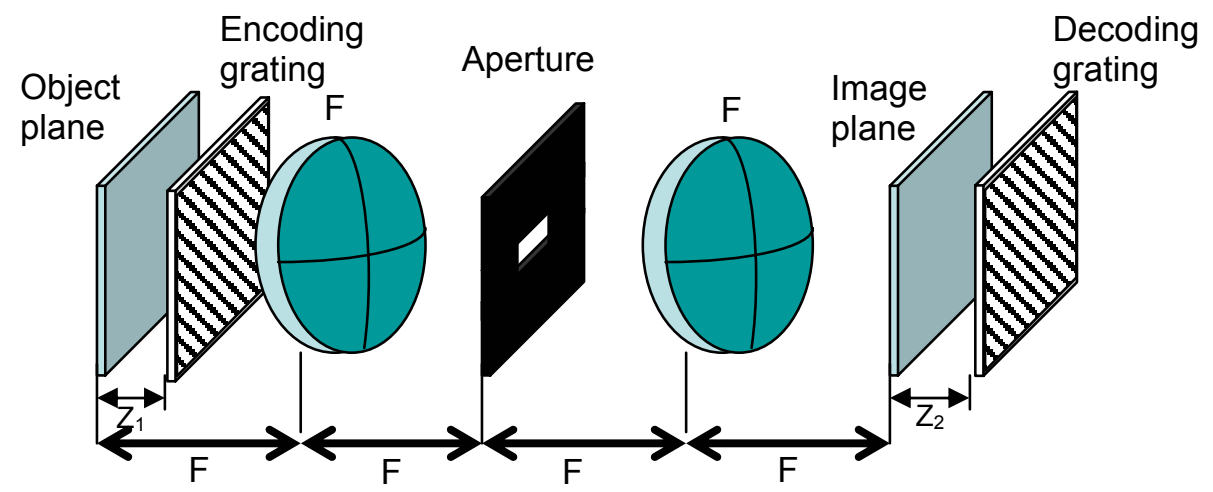

Fig. 2. Angular multiplexing basic approach.

\subsubsection{Wavelength multiplexing}

In order to apply wavelength multiplexing to have super resolved imaging one needs to have the a priori information that the object has only gray level information (it is a monochromatic object). First papers suggesting using wavelength multiplexing in order to increase resolution appear in Refs. 33-35. The approach described by Ref. 33 involves illumination of an object through a grating which causes to wavelength dispersion around the first order of diffraction. That way the various spatial features of the object are illuminated and encoded by different wavelengths. Then, a lens collects the light (performs spatial integration) and inserts it into a single mode fiber (capable of transmitting only a single degree of freedom). On the other side of the fiber the spatial information of the object is decoded using the same dispersion grating.

An improvement of this approach was proposed in Ref. 36. In that reference a $2 \mathrm{D}$ diffractive optical element allowed 2D dispersion of wavelengths and thus encoding of 2D spatial information which later on could be decoded using the same diffractive optical element.

\subsubsection{Polarization multiplexing}

In case that the object has no polarization information then by using light containing both principle polarizations one may increase the resolution by a factor of two by splitting the spatial information between the two principle polarization axes [37]. The realization of such a configuration may either be done using Wollaston prism that splits and redirects each one of the two polarizations into a different angular direction and thus can assist in coding two different spectral slots by orthogonal polarization states.

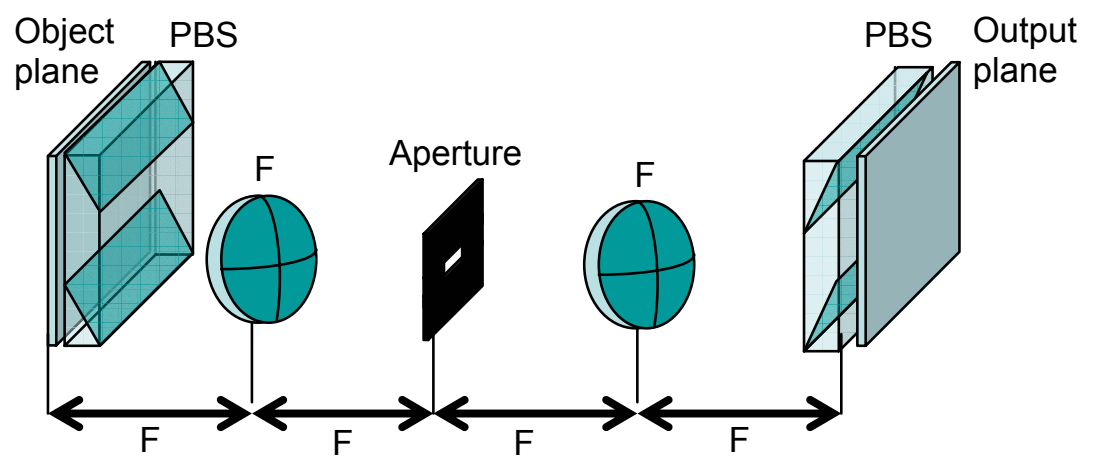

Fig. 3. Field of view increase by polarization encoding. PBS= Polarized Beam Splitter. 
Another option can be obtained using polarized beam splitters where the two different polarizations are used in order to code different regions in the field of view. It is clear that increasing the field of view while maintaining the same resolution is equivalent to maintaining the field of view and increasing the sampling resolution (as obtained in optical zooming). In the example of Fig. 3 we propose to maintain the same sampling resolution and to increase the imaged field of view by a factor of two. Since there are only two orthogonal polarization states, the increase of resolution or of the field of view may only be improved by a factor of two (in one dimension).

Another approach which combines polarization and temporal multiplexing allows improving the resolution by a larger factor as presented in Ref. 38. There, non orthogonal polarization states are used to encode plurality of spectral slots. After those slots are mixed together (when passed through the band limited imaging aperture) they are separated and the high resolution image is reconstructed by performing several sequential samplings of the output image through several non-orthogonal polarizers (e.g. by rotating a polarizer while capturing images). In this case, in contrast to the previous one, $2 \mathrm{D}$ resolution improvement may be obtained.

\subsubsection{Gray level multiplexing}

The gray levels or the dynamic range domain is another example of a domain that may be used in order to encode, transmit and then to decode the spatial information of the imaged object [39]. We assume that we have the a priori information that the dynamic range of the input object is limited. We attach a gray level coding mask to the input object. Thus, prior to the blurring due to the low resolution imaging, to each pixel of the input we attach a different transmission value while the ratio between every one of those values is $2^{\mathrm{M}}$ where $\mathrm{M}$ is the a piori known and limited number of bits spanning the dynamic range of the imaged object. Obviously, the imager should have sufficient number of dynamic range bits. It should be at least $\mathrm{M} \times \mathrm{K}^{2}$ where $\mathrm{K}$ is the super resolution improvement factor in every spatial dimension.

\subsection{Active projection techniques}

In the approaches described by the previous Sec. the super resolution was obtained by using physical components created the encoding and decoding operation. The time multiplexing technique [9] required contact between the rotating encoding grating and the object. In cases that the object is not accessible one may apply active projection techniques based upon similar operation principles.

For instance, instead of physically attaching the grating to the object one may project this grating or to generate an interference grating on the object by directing several coherent sources towards the object [14]. By modifying the relative phase of those sources the generated interference grating will move over the object and perform the Doppler coding as previously explained. The second decoding grating can be realized by digital post processing $[13,15,16]$. Instead of time integrating, the camera will capture the temporal sequence of images and then multiply each one of them by the proper decoding grating that is shifted along the frames and then it will sum together the yielded result.

Another example is the case where instead of interference grating which is a periodic function we project a random pattern such as speckles. In this case we illuminated the object through a diffuser which realizes a product between the random speckle distribution and the spatial reflectivity distribution of the object [17]. Then, we shift the diffuser which creates a projection of temporally varied random distributions. These random distributions contain small features as required and they are realized in a simple way of just illuminating a diffuser (no additional high quality lenses are required). The resolution improvement is according to the smallest feature in the random pattern.

The product is captured by the low resolution imager. Every low passed image, of the temporal sequence, is digitally multiplied by an identical decoding random distribution. After averaging the sequence of decoded images we obtain the desired 
reconstruction. The operation principle is similar to the one given for the basic time multiplexing approach [9]: the temporal variation of the random patterns allows the separation of the scrambled original spectral information of the object (multiplication in space domain between the projected pattern and the object's reflectivity equals to a convolution between those two spectral distributions).

In a similar way to the lateral movement used to perform encoding-decoding process, synchronized axial movement of two physical Ronchi gratings also allow super resolved imaging [40]. Here, both gratings are not in close contact with neither the object nor the detector and are positioned between the object and the image planes, respectively. In addition a super resolution effect may also be achieved via rotating grating (rather than moving) and digital post-processing [41]. Here, a 1-D physical grating that is placed near the input object (but not in close contact) is used to generate structured illumination onto it. The $2 \mathrm{D}$ super resolved effect is achieved by continuously rotating by 180 degrees the encoding grating and computing the output intensity in a continuous way. Finally, the decoding process is digitally performed and yields the super resolved image.

\subsection{Holographic approaches}

In summary, time multiplexing super resolution can be understood as a synthetic enlargement in the optical system aperture that improves the resolution of the imager in comparison with the resolution presented by the same optical system without applying the superresolving approach. This target can be achieved by encoding the object's spatialfrequency information into the temporal bandwidth degree of freedom. Typically, this procedure permits the passage of additional information of the object's spectrum through the limited aperture of the imaging system in time sequence, that is, as independent events. After that, a decoding is needed to rearrange the spatial-frequency information of the input object in order to obtain a high quality super resolved image.

As we have reported in Sec. 2.1, the classical encoding-decoding process is performed using diffraction gratings [9-14, 30-42]. One way to understand how this process is produced is by analyzing the encoding stage as a shift in the object's spectrum due to the action of the grating: each diffraction order down shifts the object's spectrum at the Fourier plane. This fact also happens when tilted illumination impinges on the input object. In the latter case, the shift is produced by the oblique illumination angle instead of by the diffraction angle defined in the grating's equation for each diffraction order. Figure 4 depicts the equivalence between diffraction orders of a grating a set of coherent tilted beams incoming from a set of point sources. The object's spectrum shift allows the transmission of another frequency band that falls out the limited aperture under conventional on-axis illumination. And this process is usually performed in time sequence, that is, each one of the tilted beams are shot sequentially in time in such a way that different frequency bands are transmitted at different instants.

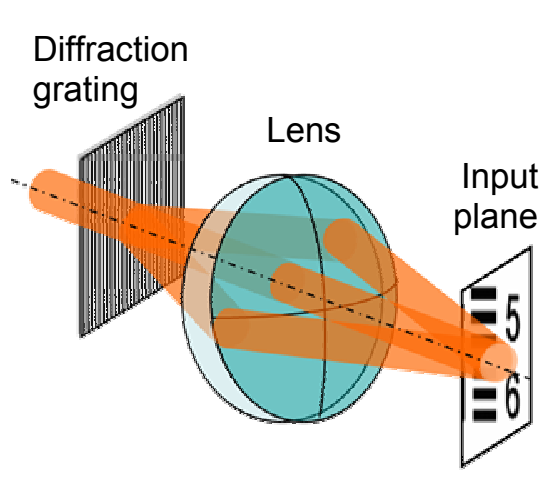

\section{Set of coherent point sources}

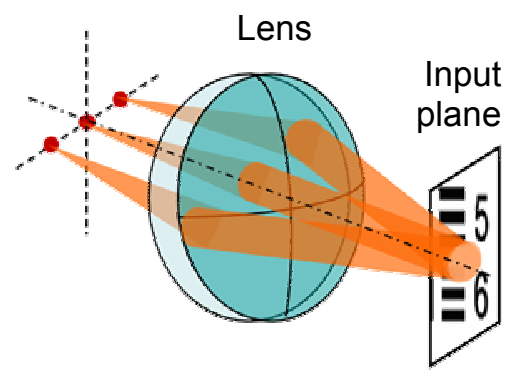

Fig. 4. Analogy between diffraction gratings (left) and tilted illumination (right). 
After its transmission through the system's aperture, this frequency band needs to be shifted back to its original position at the object's spectrum. This fact can be accomplished using holographic methods and it will be the subject under study in this Sec.

Notice that, although in a strict way the equivalence between diffraction grating and tilted beams should be considering a set of coherence point sources, the possibility that such sources will be incoherent one to each other (like in a VCSEL array) offers new capabilities to holographic approaches in superresolution imaging. This is true because the approach could be performed using time multiplexing (sequential activation of the different sources) or using spatial multiplexing (all the sources lighted on at the same time) [42].

\subsubsection{Super resolution by multi-exposed hologram}

The first attempt to achieve synthetic aperture generation from different recordings was performed in the early seventies by Stroke [43, 44] and Ueda and Sato [45]. The Stroke concept is based on the superposition of a set of small-aperture partial-frequency range component images directly in a single photographic plate. Such small apertures were obtained by masking a large aperture in order to decrease the resolution. The final results showed an image equivalent in resolution to that one obtained with the large-aperture full-frequency range optical system.

The basic idea of the Ueda and Sato method [45] implies the obtaining of a super resolved hologram in which a coherent superposition of a set of sequential images is recorded during the exposure time interval. Thus, assuming that the spatial frequency bandwidth of the optical system is narrower than that of the input object, a super resolved image will be obtained in the hologram reconstruction because each individual hologram is sequentially recorded using different tilted illumination beams onto the object and complementary off-axis reference waves at the hologram plane. Modifications of the former setup were reported later on exhibiting additional capabilities [46-48].

In fact, the approaches presented in Refs. 43-48 can be considered as the first works in time multiplexing super resolved holography. Moreover, some of them $[47,48]$ still used digital methods instead of holographic mediums as recording devices and postprocessing stages.

\subsubsection{Super resolution in digital holography}

Nowadays, digital holography is a well-known and widely-used technique that allows fast, non-destructive, full-field, high-resolution quantitative complex amplitude measurements of imaged objects. The amplitude distribution of the imaging beam is added in the hologram plane with a reference wave and the hologram is recorded by using a CCD camera. Then, the object wavefront is reconstructed numerically by simulating the back propagation of the complex amplitude using the Kirchhoff-Fresnel propagation equations. However, for both off-axis and on-axis holographic configurations, both the finite number and the size of the CCD pixels limit the resolution of the digital holographic approach.

A direct way to improve the resolution in digital holography is by generating a synthetic aperture coming from the combination of different holograms recorded at different camera positions in order to construct a larger digital hologram [49-51]. In these approaches, the resolution improvement factor is equal to the number of recorded holograms.

Recently, a new generation of methods is rising up [52, 53]. They are based on the combination of diffraction gratings and digital holographic tools. In a similar way to the Lukosz concept [9], the diffraction grating is used to down shift high order frequency bands of the object spectrum towards the imaging system (in this case a CCD device). By reinserting a reference beam at the recording plane in off-axis Fourier holographic configuration, each additional band is recovered by spatial filtering at the spatialfrequency domain. And finally, a synthetic aperture is generated by digital post- 
processing that yields in a super resolved imaging. Experimental validation for 1D [52] and 2D [53] cases have been demonstrated.

Another way to down shift high order frequency content of the object's spectrum is by using tilted illumination instead of a grating. Thus, super resolution in digital holography has been also achieved by combining angular multiplexing using tilted illumination with coherence multiplexing [54]. The resulting approach not only improves the spatial resolution of the imaged object but also can be performed in a single illumination step.

\subsubsection{Super resolution in digital holographic microscopy}

Aside the previously reported approaches, other authors had also implemented interferometric configurations to study the resolution improvement in optical imaging systems [55-59]. However, time multiplexing has been recently combined with digital holographic microscopy with the motivation of improving the limited resolving power provided by the objective lenses used in microscopy. In that sense, several super resolved approaches have been developed for different application fields such as interferometric lithography [60], scanning holographic microscopy [61] and interferometric imaging [42]. Figure 5 depicts the basic setup in a super resolution approach based on holographic tools and tilted illumination. Basically, the illumination is collimated by a lens and split by a first beam splitter (BS) allowing a Mach-Zehnder architecture. In one optical path (imaging arm) the input object and the limited aperture imaging system take place while the other optical path (reference arm) allows the holographic recording at the CCD plane after being recombined by a second BS. Tilted illumination provides the transmission of different frequency slots of the object spectrum through the limited aperture (vertical slit in Fig. 5) which can be recovered by holographic tools. Although the imaging system depicted in Fig. 5 is a 4F configuration optical system (see Ref. 42), super resolution by holographic tools can be extended to microscope lenses.

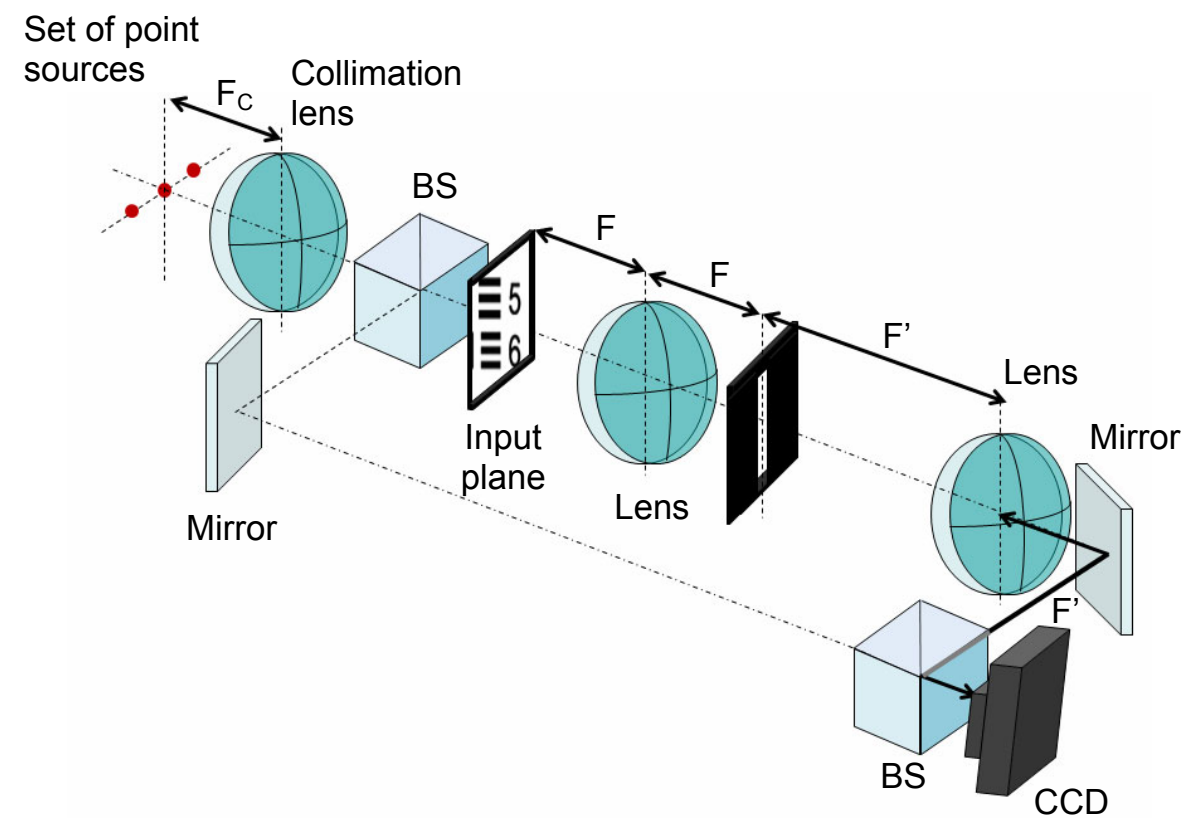

Fig. 5. Basic setup in a super resolved approach based on holographic tools.

In fact, a significant case of interferometric imaging is obtained in the field of microscopy [62-67] where synthetic aperture generation permits high-resolution images using low NA microscope lenses (typically below $0.3 \mathrm{NA}$ value). These methods are attractive due to the fact that they allow high quality imaging while maintaining the benefits regarding the use of a low NA lens, that is, long working distance, large field of 
view, large depth of focus and relatively low cost in comparison with a high aperture lens. However, they do not provide significantly high synthetic aperture values.

For this reason, other approaches [68-72] apply the same underlying principle but using medium NA lenses (ranging from 0.3 to 0.7 ). This fact permits the definition of synthetic numerical apertures higher than the theoretical value for air-immersed imaging system but with the added value of the unique feature provided by holography: the recovery of the complex amplitude distribution of the wavefront that is leaving the sample. Thus, Ref. 72 takes this advantage and presents an approach that allows super resolved imaging of 3D samples by numerical refocusing at different sample planes.

\section{GEOMETRICAL SUPER RESOLUTION}

The geometry of the detector and the shape of its pixels play a major role as well in setting the resolution limit of a given imaging system. The common way to improve the number of sampling pixels is just by using what is called a micro scanning [73, 74]. In this approach a set of images are captured while sub pixel relative shift is created between two sequential frames. The main problem of this approach is that it increases the spatial sampling density but it does not address the spatial low passing generated due to the fact that each pixel is not a delta function but rather an area that is integrating all the photonic information impinging on it.

By using proper mathematical analysis it can be shown that basically the spectrum of the object is multiplied by the Fourier transform of the spatial responsivity of a single pixel. The larger the pixel is, the more low passing the effect it yields. An inverse filtering approach may be applied in order to reduce this low pass effect [75]. The point is that the spatial responsivity of pixels usually has rectangular shape and thus its Fourier has a lot of zeros as well as low values which makes the inverse filtering very sensitive to noises. Note that first such zero would appear at twice the Nyquist frequency limit.

One proposed solution was to attach to the detector a special periodic mask (with period equals to the pitch between pixels in the sampling array) that will multiply the responsivity of each pixel and modify its spectral shape such that no zeros will be generated in the Fourier transform. That way the inverse filtering will be more efficient and tolerant to noises $[75,76]$. Another option is to project the periodic pattern that is required in order to modify the spectrum rather than to attach it to the detection array [77].

A different approach involves positioning a random mask near the detector (or in the intermediate image plane) that inserts sufficient amount of information such that the high resolution image can be reconstructed by matrix inversion operation [78]. In this case instead of modifying the responsivity of each pixel individually the effect is more global over the entire image. The reduced resolution due to the blurring may be considered in matrix representation where the amount of unknown parameters (the features of the high resolution image) is larger than the number of equations (the low resolution image). The addition of known a priori information by the proposed random mask can add more equations which make the matrix inversion feasible.

Another approach to be applied for geometrical super resolution is related to aperture coding. In this case since the resolution limiting factor is not the $\mathrm{F}$ number of the imaging lens but rather the pitch of the pixels in the detection array, all the spatial information is passed through the aperture of the lens prior to its being sampled by the detector. Thus, by placing special coding mask (spatial transmission filter) that is attached to the aperture of the lens will allow generation of orthogonal coding of the various spectral regions. Then, due to sub optimal sampling the spectrum is folded (aliasing effect) but the information can still be recovered due to the orthogonal coding that was applied in the aperture plane [79].

Although not directly related to geometry of the sensor there is a plurality of digital methods tempting to improve the image quality by digital image processing based upon various interpolation and estimation algorithms as the technique of Gerchberg [80] which obtains superresolution through error energy reduction. A summary of some of the digital based image processing super resolving algorithms appear in Refs. 81 and 82. 


\section{EXTENDING THE DEPTH OF FOCUS}

\subsection{General overview}

Extending the depth of focus (EDOF) for imaging systems was widely explored during the recent years and a large variety of technologies were developed. Defocused image yields reduction in the high spatial frequencies of the Optical Transfer Function leading to a blurred outcome.

There are various approaches of addressing the problem of defocusing by extending the depth of focus of a given optical imaging system. Some approaches involve insertion of optical element which encodes the optical wave front such that digital post processing may allow the extraction of the sharp image [83-87]. Other approaches include aperture apodization by absorptive mask [88-92] which extend the depth of focus but also result with reduced resolution and energetic throughput. Other approaches use diffractive optical phase elements such as multi focal lenses or spatially dense distributions that suffer from significant divergence of energy into regions that are not the regions of interest [93-95]. Other interesting techniques include tailoring the modulation transfer functions with high focal depth [96] and usage of logarithmic asphere lenses [97].

Different and interesting approach allowing extending the depth of focus in an alloptical way is introduced in Refs. 98 and 99. There, instead of using refractive or diffractive optics an interference effects are considered. Since it is an all-optical approach it may as well be used for ophthalmic applications such as contact lenses, spectacles and intraocular lenses (IOL). We will now focus on this type of technological solution.

\subsection{Ophthalmic applications}

The all-optical EDOF interference based technology of Refs. 98 and 99 was used to fabricate spectacles, soft contact lenses as well as intraocular lenses (IOLs) [100-104] in which the profile of the lens is such that the different spatial regions of the wave front that are passing through the eye pupil (and therefore through the contact lens/spectacle or the IOL with the engraved profile) are encoded in such a way that when they are all added together in the focal plane their interference (i.e. their addition) will generate more or less as sharp focal point as produced by the regular mono focal lens in its focal plane. However this is obtained not only in the previous axial location but rather also before as well as after the axial position of the focal plane. Thus, the effect that is used for this design is related to proper coding of the wave front passing through the various parts of the pupil [105] such that desired interference will occur around the axial position of the focal plane and such that the coding distribution has relatively large features leading to most of the energy to be directed towards zero diffraction order.

The underlying principle behind this concept is that the imaging lens is basically an interferometer device since all the rays going through the lens are summed together in the focal point (in regular interferometer only two beams are added and here there is a plurality of beams). If proper phase detour is added on top of the lens the in-phase addition can generate constructive interference pattern in a channel around the focal point and a destructive interference in the surrounding of the generated channel, leading to extended focal depth. The trade off in generating this desired interference pattern is in reduction of the contrast of the obtained image. Nevertheless the engraved profile is designed in such a way that the point spread function is wider (and thus the image has reduced contrast) but its Fourier transform contains all the spatial frequencies of the original MTF. This design constraint is important since because all the spatial information passes through, the brain can easily and quickly (after only a few seconds) adapt the contrast of the visualized image.

The engraved profile, that is designed to generate the desired interference, is a binary profile having large spatial features and small engraving depth. The maximal engraving depth is less than one micron and the spatial transversal features of the profile are as large as tens of microns. The optical element has annular-like structure (a small number of annular, it is not a Fresnel zone plate) [100]. 
The advantage of using such a profile is that since the spatial features are large the diffraction away from the zero order is very small which yields high energetic efficiency and throughput as well as reduced chromatic aberrations.

\section{RELATION TO NANOPHOTONICS AND CONCLUSIONS}

In this paper we have reviewed the recent research activity done in the field of optical super resolution. We discussed several approaches involving time, wavelength, polarization and field of view multiplexing to exceed the diffraction limit of resolution. Then, we have briefly reviewed common approaches allowing overcoming the geometrical limitation. The topic of extending the depth of focus which is related to axial resolution was discussed separately where digital as well as all-optical techniques were mentioned.

The capability to improve the resolution was obtained either by projection of special patterns or by positioning of spatial masks near the object or near the detection array (or in the intermediate image plane). Those masks often contain small features that for visible light approach the limit of half the wavelength (about $250 \mathrm{~nm}$ ). This is especially true in the case of wavelength multiplexing approach that is realized via optical diffractive elements generating the required spectral separation (that is used to encode the spatial information of the object).

In the approach performing polarization manipulation of light, sub wavelength features are required in order to deal with the vectorial nature of the field rather than its scalar approximation (when sub-wavelength features are available in the encoding/decoding super resolving mask, each polarization is affected differently by the same structure).

The techniques involving projection of encoding patterns also often contain small features that for the blue wavelength approach the size of $400 \mathrm{~nm}$. Because of that, the resolution limit of an optical imaging system could be reduced until its theoretical limit, that is, $\lambda / 2$. This $\lambda / 2$ theoretical resolution limit is due to definition of a transfer function associated with free space propagation, that is, there are spatial-frequencies that can propagate from one plane to another at a given distance (propagating waves) while others are quickly attenuated in propagation (evanescent waves). In that sense, free propagation acts as a low-pass filter over the overall spectrum of spatial-frequencies of the input object and final resolution limit is defined by the propagating waves.

However, there are other strategies to overcome this diffraction limit and achieve nano scale optical imaging. Thus, near-field scanning optical microscopy [106-110] forms an image of the sample by its scanning with an ultra sharp tip in the vicinity of the object and collecting the signal point by point. On the other hand, another family of methods based on a virtual light probe instead of a physical tip had also been developed. Stimulated emission depletion fluorescence microscopy [111-113] and saturated structured illumination microscopy [114-116] utilize a tiny light spot or line to scan the samples. Nonlinear response of the materials shrinks the light spot size or line width into deep sub-wavelength scale while achieving nano scale spatial resolution.

\section{References}

[1] Z. Zalevsky and D. Mendlovic, Optical Super Resolution, Springer, New-York (2002).

[2] E. Abbe, "Beitrage zur theorie des mikroskops und der mikroskopischen wahrnehmung," Arch. Mikrosk. Anat. 9, 413-468 (1873) [doi:10.1007/BF02956173].

[3] Z. Zalevsky, D. Mendlovic, and A. W. Lohmann, "Optical system with improved resolving power," Chapter 4 in Progress in Optics, E. Wolf Ed., Vol. XL, Elsevier, Amsterdam (1999). 
[4] A. W. Lohmann, R. G. Dorsch, D. Mendlovic, Z. Zalevsky, and C. Ferreira, "About the space bandwidth product of optical signal and systems," J. Opt. Soc. Am. A 13, 470-473 (1996) [doi:10.1364/JOSAA.13.000470].

[5] D. Mendlovic and A. W. Lohmann, "Space-bandwidth product adaptation and its applications to superresolution: fundamentals," J. Opt. Soc. Am. A 14, 558-562 (1997) [doi:10.1364/JOSAA.14.000558].

[6] D. Mendlovic, A. W. Lohmann, and Z. Zalevsky, "Space-badnwidth- product adaptation and its application for superresolution - examples," J. Opt. Soc. Am. A 14, 563-567 (1997) [doi:10.1364/JOSAA.14.000563].

[7] Z. Zalevsky, D. Memdlovic, and A. W. Lohmann, "Understanding superresolution in Wigner space," J. Opt. Soc. Am. A 17, 2422-2430 (2000) [doi:10.1364/JOSAA.17.002422].

[8] M. Francon, "Amelioration de resolution d'optique," Nuovo Cimento Suppl. 9, 283-290 (1952) [doi:10.1007/BF02903399].

[9] W. Lukosz, "Optical systems with resolving powers exceeding the classical limits," J. Opt. Soc. Am. 56, 1463-1472 (1967) [doi:10.1364/JOSA.56.001463].

[10] D. Mendlovic, A. W. Lohmann, N. Konforti, I. Kiryuschev, and Z. Zalevsky, "One dimensional superresolution optical system for temporally restricted objects," Appl. Opt. 36, 2353-2359 (1997) [doi:10.1364/AO.36.002353].

[11] D. Mendlovic, I. Kiryuschev, Z. Zalevsky, A. W. Lohmann, and D. Farkas, "Two dimensional superresolution optical system for temporally restricted objects," Appl. Opt. 36, 6687-6691 (1997) [doi:10.1364/AO.36.006687].

[12] A. W. Lohmann, W. Lukosz, J. Schwider, N. Streibl, and J. A. Thomas, "Array illuminators for the optical computer," Proc. SPIE 963, 232 (1988).

[13] A. Shemer, D. Mendlovic, Z. Zalevsky, J. Garcia, and P. G. Martinez, "Superresolving optical system with time multiplexing and computer decoding," Appl. Opt. 38, 7245-7251 (1999) [doi:10.1364/AO.38.007245].

[14] A. Shemer, Z. Zalevsky, D. Mendlovic, N. Konforti, and E. Marom, "Time multiplexing superresolution based on interference grating projection," Appl. Opt. 41, 7397-7404(2002) [doi:10.1364/AO.41.007397].

[15] F. Lanni, D. L. Taylor, and B. Bailey, "Field synthesis and optical subsectioning for standing wave microscopy, " US Patent 6,055,097 (1998).

[16] R. Heintzmann and C. Cremer, "Lateral modulated excitation microscopy: Improvement of resolution by using a diffraction grating," Proc. SPIE 3568, 185-196 (1999) [doi:10.1117/12.336833].

[17] J. Garcia, Z. Zalevsky, and D. Fixler, "Synthetic aperture superresolution by speckle pattern projection," Opt. Express 13, 6073-6078 (2005) [doi:10.1364/OPEX.13.006073].

[18] D. Fixler, J. Garcia, Z. Zalevsky, A. Weiss, and M. Deutsch, "Speckle random coding for 2-D super resolving fluorescent microscopic imaging," Micron 38, 121-128 (2007) [doi:10.1016/j.micron.2006.07.008].

[19] M. A. A. Neil, R. Juskaitis, and T. Wilson, "Method of obtaining optical sectioning by using structured light in a conventional microscope," Opt. Lett. 22, 1905-1907 (1997) [doi:10.1364/OL.22.001905].

[20] C. Ventalon and J. Mertz, "Quasi-confocal fluorescence sectioning with dynamic speckle illumination," Opt. Lett. 30, 3350-3352 (2005) [doi:10.1364/OL.30.003350].

[21] C. Ventalon and J. Mertz, "Dynamic speckle illumination microscopy with translated versus randomized speckle patterns," Opt. Express 14, 7198-7209 (2006) [doi:10.1364/OE.14.007198].

[22] Z. Zalevsky, J. Garcia, and C. Ferreira, "Superresolved imaging of remote moving targets," Opt. Lett. 31, 586-588 (2006) [doi:10.1364/OL.31.000586].

[23] Z. Zalevsky, E. Saat, S. Orbach, V. Mico, and J. Garcia, "Exceeding the resolving imaging power using environmental conditions," Appl. Opt. 47, A1-A6 (2008) [doi:10.1364/AO.47.0000A1]. 
[24] Z. Zalevsky, S. Rozental, and M. Meller, "Usage of turbulence for super resolved imaging," Opt. Lett. 32, 1072-1074 (2007) [doi:10.1364/OL.32.001072].

[25] Z. Zalevsky, J. Garcia, P. Garcia-Martinez, and C. Ferreira, "Spatial information transmission using orthogonal mutual coherence coding," Opt. Lett. 20, 28372839 (2005) [doi:10.1364/OL.30.002837].

[26] V. Mico, J. García, C. Ferreira, D. Sylman, and Z. Zalevsky, "Spatial information transmission using axial temporal coherence coding," Opt. Lett. 32, 736-738 (2007) [doi:10.1364/OL.32.000736].

[27] W. Lukosz, "Optical systems with resolving powers exceeding the classical limits II,” J. Opt. Soc. Am. 57, 932-41 (1967) [doi:10.1364/JOSA.57.000932].

[28] M. A. Grim and A. W. Lohmann, "Superresolution image for one-dimensional objects," J. Opt. Soc. Am. 56, 1151-1156 (1966) [doi:10.1364/JOSA.56.001151].

[29] E. Sabo, Z. Zalevsky, D. Mendlovic, N. Konforti, and I. Kiryuschev, "Superresolution optical system using two fixed generalized Dammann gratings," Appl. Opt. 39, 5318-5325 (2000) [doi:10.1364/AO.39.005318].

[30] Z. Zalevsky, D. Mendlovic, and A. W. Lohmann, "Super resolution optical systems for object with finite sizes," Opt. Commun. 163, 79-85 (1999) [doi:10.1016/S0030-4018(99)00121-2].

[31] E. Sabo, Z. Zalevsky, D. Mendlovic, N. Konforti, and I. Kiryuschev, "Superresolution optical system using three fixed generalized gratings: Experimental results," J. Opt. Soc. Am. A 18, 514-520 (2001) [doi:10.1364/JOSAA.18.000514].

[32] J. García, V. Micó, D. Cojoc, and Z. Zalevsky, "Full field of view superresolution imaging based on two static gratings and white light illumination," Appl. Opt. 47, 3080-3087 (2008) [doi:10.1364/AO.47.003080].

[33] A. I. Kartashev, "Optical systems with enhanced resolving power," Opt. Spectrosc. 9, 204-206 (1960).

[34] J. D. Armitage, A. W. Lohmann, and D. P. Parish, "Superresolution image forming systems for objects with restricted lambda dependence" Jpn. J. Appl. Phys. 4, 273-275 (1965).

[35] S. A. Alexandrov and D. D. Sampson, "Spatial information transmission beyond a system's diffraction limit using optical spectral encoding of the spatial frequency," J. Opt. A: Pure Appl. Opt. 10, 025304 (2008) [doi:10.1088/1464$\underline{4258 / 10 / 2 / 025304] .}$.

[36] D. Mendlovic, J. Garcia, Z. Zalevsky, E. Marom, D. Mas, C. Ferreira, and A. W. Lohmann, "Wavelength multiplexing system for a single mode image transmission," Appl. Opt. 36, 8474-8480 (1997) [doi:10.1364/AO.36.008474].

[37] A. W. Lohmann and D. Paris, "Superresolution for nonbirefringent objects," J. Opt. Soc. Am. 3, 1037-1043 (1964) [doi:10.1364/AO.3.001037].

[38] A. Zlotnik, Z. Zalevsky, and E. Marom, "Superresolution with nonorthogonal polarization coding," Appl. Opt. 44, 3705-3715 (2005) [doi:10.1364/AO.44.003705].

[39] Z. Zalevsky, P. García-Martínez, and J. García, "Superresolution using gray level coding," Opt. Express 14, 5178-5182 (2006) [doi:10.1364/OE.14.005178].

[40] Z. Zalevsky, J. García, and V. Micó "Transversal superresolution with noncontact axial movement of periodic structures," J. Opt. Soc. Am. A 24, 32203225 (2007) [doi:10.1364/JOSAA.24.003220].

[41] V. Mico, Z. Zalevsky, O. Limon, and J. García, "Transverse resolution improvement using rotating-grating time-multiplexing approach," J. Opt. Soc. Am. A 25, 1115-1129 (2008) [doi:10.1364/JOSAA.25.001115].

[42] V. Mico, Z. Zalevsky, P. G. Martínez, and J. García, "Single-step superresolution by interferometric imaging," Opt. Express 12, 2589-2596 (2004) [doi:10.1364/OPEX.12.002589].

[43] G. W. Stroke, "Synthesis of large-aperture optics by successive exposure of a single photographic plate through successively placed small-aperture optics: Part 
I. Theory," Opt. Commun. 1, 283-286 (1970) [doi:10.1016/0030-4018(70)901112].

[44] G. W. Stroke, "Synthesis of large-aperture optics by successive exposure of a single photographic plate through successively placed small-aperture optics: Part II. Experimental verification," Opt. Commun. 1, 287-290 (1970) [doi:10.1016/0030-4018(70)90112-4].

[45] M. Ueda and T. Sato, "Superresolution by holography," J. Opt. Soc. Am. 61, 418-419 (1971) [doi:10.1364/JOSA.61.000418].

[46] M. Ueda, T. Sato, and M. Kondo, "Superresolution by multiple superposition of image holograms having different carrier frequencies," Opt. Acta 20, 403-410 (1973) [doi:10.1080/713818783].

[47] T. Sato, M. Ueda, and G. Yamagishi, "Superresolution microscope using electrical superposition of holograms," Appl. Opt. 13, 406-408 (1974) [doi:10.1364/AO.13.000406].

[48] T. Sato, M. Ueda, and T. Ikeda, "Real time superresolution by means of an ultrasonic light diffractor and TV system," Appl. Opt. 13, 1318-1321 (1974) [doi:10.1364/AO.13.001318].

[49] F. Le Clerc, M. Gross, and L. Collot, "Synthetic aperture experiment in the visible with on-axis digital heterodyne holography," Opt. Lett. 26, 1550-1552 (2001) [doi:10.1364/OL.26.001550].

[50] J. H. Massig, "Digital off-axis holography with a synthetic aperture," Opt. Lett. 27, 2179-2181 (2002) [doi:10.1364/OL.27.002179].

[51] R. Binet, J. Colineau, and J-C. Lehureau, "Short-range synthetic aperture imaging at $633 \mathrm{~nm}$ by digital holography," Appl. Opt. 41, 4775-4782 (2002) [doi:10.1364/AO.41.004775].

[52] Ch. Liu, Z. Liu, F. Bo, Y. Wand, and J. Zhu, "Super-resolution digital holographic imaging method," Appl. Phys. Lett. 81, 3143-3145 (2002) [doi:10.1063/1.1517402].

[53] M. Pateruzo, F. Merola, S. Grili, S. de Nicola, A. Finizio, and P. Ferraro, "Superresolution in digital holography by a two-dimensional dynamic phase grating," Opt. Express 16, 17107-17118 (2008) [doi:10.1364/OE.16.017107].

[54] C. Yuan, H. Zhai, and H. Liu, "Angular multiplexing in pulsed digital holography for aperture synthesis," Opt. Lett. 33, 2356-2358 (2008) [doi:10.1364/OL.33.002356].

[55] E. N. Leith, D. Angell, and C. P. Kuei, "Superresolution by incoherent-tocoherent conversion," J. Opt. Soc. Am. A 4, 1050-1054 (1987) [doi:10.1364/JOSAA.4.001050].

[56] H. Dupoisot, A. Poletaeff, G. Daury, and P. de Vernejoul "Off-axis recording and synthetic aperture imaging in light microscopy," Opt. Comm. 72, 42-46, (1989) [doi:10.1016/0030-4018(89)90253-8].

[57] E. N. Leith, "Small aperture, high resolution, two channel imaging system," Opt. Lett. 15, 885-887 (1990) [doi:10.1364/OL.15.000885].

[58] P. C. Sun and E. N. Leith, "Superresolution by spatial-temporal encoding methods," Appl. Opt. 31, 4857-4862 (1992) [doi:10.1364/AO.31.004857].

[59] K. Mills, Z. Zalevsky, and E. Leith, "Holographic generalized first-arriving light approach for resolving images viewed throughout a scattering medium," Appl. Opt. 41, 2116-2121 (2002) [doi:10.1364/AO.41.002116].

[60] X. Chen and S. R. J. Brueck, "Imaging interferometric lithography: Approaching the resolution limits of optics," Opt. Lett. 24, 124-126 (1999) [doi:10.1364/OL.24.000124].

[61] G. Indebetouw, Y. Tada, J. Rosen, and G. Brooker, "Scanning holographic microscopy with resolution exceeding the Rayleigh limit of the objective by superposition of off-axis holograms," Appl. Opt. 46, 993-1000 (2007) [doi:10.1364/AO.46.000993]. 
[62] V. Mico, Z. Zalevsky, P. García-Martínez, and J. García, "Superresolved imaging in digital holography by superposition of tilted wavefronts," Appl. Opt. 45, 822-828 (2006) [doi:10.1364/AO.45.000822].

[63] V. Mico, Z. Zalevsky, P. García-Martínez, and J. García, "Synthetic aperture superresolution with multiple off-axis holograms," J. Opt. Soc. Am. A 23, 31623170 (2006) [doi:10.1364/JOSAA.23.003162].

[64] V. Mico, Z. Zalevsky, and J. García, "Superresolution optical system by common-path interferometry," Opt. Express 14, 5168-5177 (2006) [doi:10.1364/OE.14.005168].

[65] S. A. Alexandrov, T. R. Hillman, T. Gutzler, and D. D. Sampson, "Synthetic aperture Fourier holographic optical microscopy," Phy. Rev. Lett. 97, 168102 (2006) [doi:10.1103/PhysRevLett.97.168102].

[66] V. Mico, Z. Zalevsky, and J. García, "Synthetic aperture microscopy using offaxis illumination and polarization coding," Opt. Commun. 276, 209-217 (2007) [doi:10.1016/j.optcom.2007.04.020].

[67] V. Mico, Z. Zalevsky, and J. García, "Common-path phase-shifting digital holographic microscopy: a way to quantitative imaging and superresolution," Opt. Comm. 281, 4273-4281 (2008) [doi:10.1016/j.optcom.2008.04.079].

[68] Ch. J. Schwarz, Y. Kuznetsova, and S. R. Brueck, "Imaging inteferometric microscopy," Opt. Lett. 28, 1424-1426 (2003) [doi:10.1364/OL.28.001424].

[69] J. R. Price, P. R. Bingham, and C. E. Thomas Jr. "Improving resolution in microscopic holography by computationally fusing multiple, oblique illuminated object waves in the Fourier domain," Appl. Opt. 46, 827-833 (2007) [doi:10.1364/AO.46.000827].

[70] Y. Kuznetsova, A. Neumann, and S. R. J. Brueck "Imaging interferometric microscopy - approaching the limits of optical resolution," Opt. Express 15, 6651-6663 (2007) [doi:10.1364/OE.15.006651].

[71] A. Neumann, Y. Kuznetsova, and S. R. Brueck, "Structured illumination for the extension of imaging interferometric microscopy," Opt. Express 16, 6785-6793 (2008) [doi:10.1364/OE.16.006785].

[72] V. Mico, Z. Zalevsky, C. Ferreira, and J. García, "Superresolution digital holographic microscopy for three-dimensional samples," Opt. Express 16, 19261-19270 (2008) [doi:10.1364/OE.16.019260].

[73] J. Fortin, P. Chevrette, and R. Plante "Evaluation of the microscanning process," Proc. SPIE 2269, 271-279 (1994) [doi:10.1117/12.188643].

[74] S. Borman and R. Stevenson, "Super-resolution from image sequences - A review," Proc. Midwest Symposium on Circuits and Systems, 374-378 (1998) [doi:10.1109/MWSCAS.1998.759509].

[75] Z. Zalevsky, D. Mendlovic, and E. Marom, "Special sensor masking for exceeding system geometrical resolving power," Opt. Eng. 39, 1936-1942 (2000) [doi:10.1117/1.602578].

[76] Z. Zalevsky, N. Shamir, and D. Mendlovic, "Geometrical super-resolution in infrared sensor: Experimental verification,” Opt. Eng. 43, 1401-1406 (2004) [doi:10.1117/1.1737372].

[77] D. Fixler, J. Garcia, Z. Zalevsky, A. Weiss, and M. Deutsch, "Pattern projection for subpixel resolved imaging in microscopy," Micron 38, 115-120 (2007) [doi:10.1016/j.micron.2006.07.007].

[78] A. Borkowski, Z. Zalevsky, and B. Javidi, "Geometrical super resolved imaging using non periodic spatial masking," J. Opt. Soc. Am. A 26, 589-601 (2009).

[79] J. Solomon, Z. Zalevsky, and D. Mendlovic, "Geometrical superresolution using code division multiplexing," Appl. Opt. 42, 32-40 (2005) [doi:10.1364/AO.44.000032].

[80] R. W. Gerchberg, "Super-resolution through error energy reduction," J. Mod. Opt. 21, 709-720 (1974) [doi:10.1080/713818946].

[81] S. C. Park, M. K. Park, and M. G. King, "Super-resolution image reconstruction: a technical review," IEEE Signal Processing Magazine 20, 21-36 (2003). 
[82] S. Farsiu, D. Robinson, M. Elad, and P. Milanfar, "Advances and challenges in super-resolution," Int. J. Imaging Sys. Technol. 14, 47057 (2004) [doi:10.1002/ima.20007].

[83] J. van der Gracht, E. Dowski, M. Taylor, and D. Deaver, "Broadband behavior of an optical-digital focus-invariant system," Opt. Lett. 21, 919-921 (1996) [doi:10.1364/OL.21.000919].

[84] W. T. Cathy and E. R Dowski, "Extended depth of field optical systems," PCT publication WO 99/57599 (November 1999).

[85] W. T. Cathy, "Extended depth field optics for human vision," PCT publication WO 03/052492 (June 2003).

[86] W. T. Cathy and E. R Dowski, "Apparatus and method for extending depth of field in image projection system," US patent 6069738 (May 2000).

[87] E. R Dowski and W. T. Cathey, "Extended depth of field through wave-front coding," Appl. Opt. 34, 1859-1866 (1995) [doi:10.1364/AO.34.001859].

[88] J. O. Castaneda and L. R. Berriel-Valdos, "Zone plate for arbitrary high focal depth," Appl. Opt. 29, 994-997 (1990).

[89] C. M. Hammond, "Apparatus and method for reducing imaging errors in imaging systems having an extended depth of field," US patent 6097856 (August 2000).

[90] N. Atebara and D. Miller, "Masked intraocular lens and method for treating a patient with cataracts," US patent 4955904 (September 1990).

[91] D. Miller and E. Blanko, "System and method for increasing the depth of focus of the human eye," US patent 6554424 (April 2003).

[92] J. O. Castaneda, E. Tepichin, and A. Diaz, "Arbitrary high focal depth with a quasi optimum real and positive transmittance apodizer," Appl. Opt. 28, 26662669 (1989).

[93] E. Ben-Eliezer, E. Marom, N. Konforti, and Z. Zalevsky, "Experimental realization of an imaging system with an extended depth of field," Appl. Opt. 44, 2792-2798 (2005) [doi:10.1364/AO.44.002792].

[94] E. Ben-Eliezer, Z. Zalevsky, E. Marom, and N. Konforti, “All-optical extended depth of field imaging system," J. Opt. A: Pure Appl. Opt. 5, S164-S169 (2003) [doi:10.1088/1464-4258/5/5/359].

[95] E. Ben-Eliezer, Z. Zalevsky, E. Marom, N. Konforti, and D. Mendlovic, “All optical extended depth-of field imaging system," US patent No. 7158317.

[96] A. Sauceda and J. Ojeda-Castaneda, "High focal depth with fractional-power wavefronts," Opt. Lett. 29, 560-562 (2004) [doi:10.1364/OL.29.000560].

[97] W. Chi and N. George, "Electronic imaging using a logarithmic asphere," Opt. Lett. 26, 875-877 (2001) [doi:10.1364/OL.26.000875].

[98] Z. Zalevsky, A. Shemer, A. Zlotnik, E. Ben-Eliezer, and E. Marom, “All-optical axial super resolving imaging using low-frequency binary-phase mask," Opt. Express 14, 2631-2643 (2006) [doi:10.1364/OE.14.002631].

[99] I. Raveh and Z. Zalevsky, "All-optical axially multi-regional super resolved imaging," Opt. Express 15, 17912-17921 (2007) [doi:10.1364/OE.15.017912].

[100] Z. Zalevsky, S. Ben Yaish, O. Yehezkel, and M. Belkin, "Thin spectacles for myopia, presbyopia and astigmatism insensitive vision," Opt. Express 15, 10790-10803 (2007) [doi:10.1364/OE.15.010790].

[101] O. Yehezkel, S. Ben-Yaish, A. Zlotnik, M. Belkin, and Z. Zalevsky, "A novel myopia correcting lens which reduces the need for accommodation for near vision tasks," Association for Research in Vision and Optholmology (ARVO), Florida, USA, (April 2008).

[102] S. Ben Yaish, A. Zlotnik, O. Yehezkel, M. Belkin, and Z. Zalevsky, "Omni-focal refractive correction lens: A potential substitute for bi/multi-focal lenses," Association for Research in Vision and Optholmology (ARVO), Florida, USA (April 2008).

[103] A. Zlotnik, S. Ben Yaish, O. Yehezkel, M. Belkin, and Z. Zalevsky, "Thin films as spectacles and contact lenses for aberration-corrected vision via brain adaptation to contrast," J. Vision 8, 263 (2008). 
[104] S. Ben Yaish, A. Zlotnik, I. Raveh, O. Yehezkel, M. Belkin, K. Lahav, and Z. Zalevsky, "Omni-focal refractive focus correction technology as a substitute for bi/multi-focal intraocular lenses, contact lenses, and spectacles," Proc. SPIE 7163 (2009) [doi:10.1117/12.807349].

[105] C. Cogswell, "Wavefront coding extends the depth of field in high numerical aperture brightfield, phase and interference contrast microscopy," Integrated Computational Imaging Systems, OSA Technical Digest Series, paper ITuB2 (2001).

[106] E. Betzig, J. K. Trautman, T. D. Harris, J. S. Weiner, and R. K. Kostelak, "Breaking the diffraction barrier: optical microscopy on nanometric scale," Science 251, 1468-1470 (1991) [doi:10.1126/science.251.5000.1468].

[107] E. Betzig and J. K. Trautman, "Near-field optics: microscopy, spectroscopy, and surface modification beyond the diffraction limit," Proc. Natl. Acad. Sci. USA 257, 189-195 (1992) [doi:10.1126/science.257.5067.189].

[108] D. Courjon, Near-field microscopy and near-field optics, Imperial College Press, London (2003).

[109] D. P. Sai and W. C. Lin, "Probing the near fields of the super-resolution near field optical structure," Appl. Phys. Lett. 77, 1413-1415 (2000) [doi:10.1063/1.1290692].

[110] E. A. Ash and G. Nocholls, "Super-resolution aperture scanning microscope," Nature 237, 510-512 (1972) [doi:10.1038/237510a0].

[111] S. W. Hell and J. Wichmann, "Breaking the diffraction resolution limit the stimulated emission: stimulated emission depletion microscopy," Opt. Lett. 19, 780-782 (1994) [doi:10.1364/OL.19.000780].

[112] S. W. Hell, "Toward fluorescence microscopy," Nat. Biotechnol. 21, 1347-1355 (2003).

[113] G. Donnert, J. Keller, R. Medda, M. A. Andrei, S. O. Rizzoli, R. Lührmann, R. Janh, Ch. Eggeling, and S. W. Hell, "Macromolecular-scale resolution in biological fluorescence microscopy," Proc. Natl. Acad. Sci. USA 103, 1144011445 (2006) [doi:10.1073/pnas.0604965103].

[114] R. Heintzmann, T. M. Jovin, and C. Cremer, "Saturated patterned excitation microscopy - a concept for optical resolution improvement," J. Opt. Soc. Am. A 19, 1599-1609 (2002) [doi:10.1364/JOSAA.19.001599].

[115] R. Heintzmann, "Saturated patterned excitation microscopy with twodimensional excitation patterns," Micron 34, 283-291 (2003) [doi:10.1016/S0968-4328(03)00053-2].

[116] M. G. L. Gustafsson, "Nonlinear structured illumination microscopy: wide-field fluorescence imaging with theoretically unlimited resolution," Proc. Natl. Acad. Sci. USA 102, 13081-13086 (2005) [doi:10.1073/pnas.0406877102]. 